Y-12

OAK RIDGE

Y-12

PLANT

\title{
OVERVIEW OF NWIS SOFTWARE
}

\author{
J. A. Mullens
}

Nuclear Materials Management and Storage Program Office

January 2000

Prepared by the Oak Ridge Y-12 Plant

Oak Ridge, Tennessee 37831 managed by

Lockheed Martin Energy Systems, Inc. for the 


\section{DISCLAIMER}

This report was prepared as an account of work sponsored by an agency of the United States government. Neither the United States Government nor any agency thereof, nor any of their employees, makes any warranty, express or implied, or assumes any legal liability or responsibility for the accuracy, completeness, or usefulness of any information, apparatus, product, or process disclosed, or represents that its use would not infringe privately owned rights. Reference herein to any specific commercial product, process, or service by trade name, trademark, manufacturer, or otherwise, does not necessarily constitute or imply its endorsement, recommendation, or favoring by the United States Government or any agency thereof. The views and opinions of authors expressed herein do not necessarily state or reflect those of the United States Government or any agency thereof. 


\title{
Overview of NWIS Software
}

\author{
James A Mullens \\ Oak Ridge National Laboratory \\ January 2000
}

\section{Introduction to Nuclear Weapons Identification System}

The Nuclear Weapons Identification System (NWIS) is a system that performs radiation signature measurements on objects such as nuclear weapons components. NWIS consists of a ${ }^{252} \mathrm{Cf}$ fission source, radiation detectors and associated analog electronics, data acquisition boards, and a computer running Windows NT and the application software. NWIS uses signal processing techniques to produce a radiation signature from the radiation emitted from the object. This signature can be stored and later compared to another signature to determine whether two objects are similar. A library of such signatures can be used to identify objects in closed containers as well as determine attributes such as fissile mass and in some cases enrichment.

NWIS uses a ${ }^{252} \mathrm{Cf}$ source on one side of the object to produce radiation that its detectors measure on the other side of the target (active mode). If the object naturally emits enough radiation, the ${ }^{252} \mathrm{Cf}$ source is not required (passive mode).

The NWIS data acquisition hardware has five detector channels. Each channel receives shaped detector pulses and times those pulses with 1 nanosecond resolution. In active mode measurements one of these channels receives pulses from a detector measuring the ${ }^{252} \mathrm{Cf}$ source fissions. Thus, for active mode measurements, NWIS has the time of each ${ }^{252} \mathrm{Cf}$ fission and the subsequent injection of neutrons and gamma rays into the object. The remaining channels receive pulses from the detectors measuring radiation from the object. These detectors record the amount and time of radiation exiting the object. By correlating the radiation events among the source and the other detectors, and among the detectors themselves, a characteristic response of the object to ${ }^{252} \mathrm{Cf}$ radiation or its own internal radiation is measured.

The data acquisition hardware consists of two custom-made boards. The Data Capture and Compression (DCC) board is built around a Gallium Arsine (GaAs) chip designed at ORNL. This chip assigns a time to each pulse received on the five detector channels and passes five compressed streams of time stamp data to the Data Acquisition (DA) board. The DA board performs additional data compression, consolidates the five data streams into one, formats the data, and passes it across the computer's PCl bus into computer memory. The computer processor performs the signal processing required to calculate the signatures and saves the result to disk. Signature analysis software provides the means to manipulate and match signatures in a signature library. 


\section{Executables}

There are three executables built from the software:

1. Windows NT kernel-mode device driver,

2. data acquisition application, and

3. data analysis application.

The device driver is the interface between the NWIS data acquisition boards and the remainder of the software. The data acquisition executable is the user's tool for making an NWIS measurement; it has limited data display abilities. The data analysis executable is user's tool for displaying and analyzing an NWIS measurement, including matching it to other NWIS measurements.

Normally, the NWIS user has full access to the NWIS measurement data. However, both the data acquisition and data display executables can be built so as to limit the computer displays to forms that do not reveal classified information. These special builds are used during demonstrations to visitors when the demonstration involves classified measurements, as discussed below.

\section{Data Acquisition Software}

NWIS detector data consist of the time that every detector registered a radiation count. The hardware monitors the detector signal channel during each sampling interval and produces a time stamp representing that interval whenever a count occurs. The sampling interval is typically one nanosecond. Count rates vary with the ${ }^{252} \mathrm{Cf}$ source strength, and are typically kept below 2 million counts per second (cps), so fewer than 1 in 500 sampling intervals registers a count.

In addition to the five physical detector channels, the user can define a "combined detector channel". The user selects a group of detectors to be combined as if they were a single detector: all counts from all of the detectors go into a synthesized "combined detector" and are processed as if they were an additional signal channel. This feature is used the multiplicity measurements.

The amount of detector data collected during a run is too large to store permanently. For example, at 2 million cps on each channel this data would require 10 gigabytes of storage for a 500-second measurement. Instead, NWIS calculates signatures from the time stamps during data acquisition, then discards the time stamps.

The Data Acquisition User Interface (DAUI) is the application software that controls signature measurement. DAUI provides a GUI used to configure, run, and store the measurement.

The NWIS measurement is an application of signal processing techniques for random processes (radiation). Measurement configuration options include number of detectors, sample rate, length of measurement, signature displays, and other parameters. A typical configuration is five detectors, $10^{9}$ samples per second, $10^{9}$ data blocks, and a data block length of $512 \mathrm{~ns}$ (a total of 512 seconds of data). The user can configure DAUl to display a variety of signatures during the measurement in order to monitor the progress of the measurement. 
The measurement is made in "loops". A loop is one complete cycle of data acquisition and data display, and a complete measurement consists of one or more loops. NWIS will acquire up to $10^{8}$ data blocks in one loop, then update the measurement display. DAUI is designed to run unattended but can be paused or halted during the run, at the end of any loop.

When the measurement is complete the user can save the signature to a disk file and add it to a signature catalog for subsequent analysis.

No attempt is made to store the radiation detector count time stamps. Instead, DAUI uses them to produce the following while the data is being acquired, then discards the time stamps:

1. auto and cross correlations,

2. count probability distributions for each detector and one combination of detectors (not including the Cf source detector).

3. a set of triggered count probability distributions in which each signal channel is a trigger and the corresponding counting channel is the (synthesized) combined detector.

All auto and cross correlations are calculated (ten correlations for five signals) using an algorithm which takes advantage of the fact that very few sampling intervals contain a detector count. The count probability distribution distributions (PDFs) are simply the probabilities of observing $\mathrm{N}$ counts within a data block (i.e., a fixed period of time, typically $512 \mathrm{~ns}$ ), so no data processing of any type is required beyond recording how many data blocks contained $\mathrm{N}$ detector counts. The triggered count probability distributions are the probabilities of observing $\mathrm{N}$ counts in the combined detector channel after a count in the trigger channel. The counting period for the triggered count is also the length of one data block.

DAUI measures the correlations and count probabilities, averaging over a time period set by the user, and writes the average values to a computer disk file.

DAUI does not perform any processing of the data based on the data content, except that a total absence of counts from some detectors is noted because this suggests a detector failure. DAUI is written as a general-purpose program. It is not written to discern the nature of the object being measured or perform any processing dependent on its nature.

The software discards the first data block acquired because the board returns incorrect times for counts in the first block. This occurs because the DCC is being reset and takes some time to establish proper operation at $1 \mathrm{GHz}$. The software also discards counts in adjacent $1 \mathrm{~ns}$ time bins. It is physically impossible for counts to occur in adjacent $1 \mathrm{~ns}$ time bins since the count pulse width is at least $3 \mathrm{~ns}$. This is a small timing problem on the DCC chip, which causes a pulse coming at the end of a 1 ns sampling interval to be placed in two time bins. This timing problem occurs only when the DCC is operating at 1 $\mathrm{GHz}$.

While a measurement is progressing, DAUI can calculate and display correlations, PDFs, and other quantities derived from them using well-known signal processing techniques. These quantities include the auto and cross spectra, coherences, and ratio of spectral densities. 
The software interface to the NWIS data acquisition boards is a Windows NT kernel-mode device driver, written at ORNL. The device driver provides two types of functions. First, board I/O port access functions allow the application software to directly control the NWIS boards when necessary. Second, bus-master DMA transfers allow the application software to read the time stamp data stream at rates up to $44 \mathrm{Mb} / \mathrm{s}$ (the $\mathrm{l} / \mathrm{O}$ limit of the NWIS board). DA simply transfers bytes from the NWIS data acquisition boards to the computer. It is not written to decode the detector data, perform any calculation with the data, or otherwise take any action based on the contents of the data stream.

\section{Interactive Data Analysis Software}

IDAS is the user's data analysis software. It reads and operates on the measurement data stored by DAUI. IDAS performs two types of processing.

The first type of processing is calculating and displaying the same group of signal processing quantities that DAUI calculates. (IDAS and DAUI share the software that does this). These quantities are calculated using standard signal processing techniques. IDAS adds "ratio plots" to the standard set. A ratio plot is intended to show how two measured objects match by displaying the ratio of their measurements; processing consists of simply dividing a quantity measured for one object by the same quantity measured for another object. The measurement in the numerator typically represents an "unknown object" while the measurement in the denominator typically represents a "reference object". When multiple reference measurements are used, IDAS adds "ratio bounds" to the plot. These bounds are calculated by finding the minimum and maximum ratios among the reference measurements, displaying them along with the ratio of the unknown measurement (so that an unknown that matches the references will lie within the bounds).

The second type of processing is a form of measurement identification called template matching. This function matches an "unknown" to a "reference" measurement. The reference measurement is simply some measurement specified by the user: IDAS does not keep its own library of reference measurements. This match is a statistical comparison of two measurements, where the statistical uncertainty involved stems from the fundamentally random nature of the radiation signals measured. The statistical comparison makes use of standard signal processing techniques to estimate the uncertainties in the measurement, and standard statistical techniques to make the comparisons. The software allows the user to exclude portions of the NWIS measurement, and allows the user to setup some statistical criteria for the matching performed. This processing is intended to be generic in that it can be used with any measured object. It does not incorporate any knowledge of weapons. It is intended to be generic, and not to assume any particular type of measured object when performing the matching.

\section{Installation}

The device driver is installed by running INSTDRV.EXE. The command is:

INSTDRV DA0 < full path name of the driver file DA.SYS>

From the Control Panel's Devices utility, the driver startup should be set to Automatic.

The GraphiC library needs to have an environmental variable GPCWIN set to the GraphiC directory. Use the Control Panel's System utility. 


\section{IDAS User Manual}

For IDAS version 0.8.12

James A Mullens

January 2000 


\section{IDAS (ANWIS1) ViewS}

There are two views (windows) available: a graphical and a text view. The graphical view plots the measurement data and is the main view of the application. Most menu bar commands are active when the graphical view is active. The text view shows measurement summary information or a table of the data in the plot view. Most menu bar commands are not active when the text view is active.

The user switches between the windows using the menu bar Window selection.

\section{IDAS (ANWIS1) Commands}

File

Open

Open a measurement (PAR file) or a measurement catalog (CAT file).

\section{Close}

Close the currently open measurement or catalog. Since the GraphiC library provides for only one open graphics window, the current measurement must be closed before a new measurement can be opened.

Save

This command is disabled to indicate that the user cannot overwrite a measurement file.

\section{SaveAs}

This command is enabled when the text view is active. The contents of the text view are saved to a text file selected by the user.

Print

Print the active window (text or graphics).

\section{Print Setup}

Select printer options such as page orientation (landscape or portrait). IDAS automatically requests the user's printer setup for the first print. This is done through a GraphiC function (DoPrintSetup). Printer setup can be done only once due to some unknown interaction between IDAS, GraphiC, and/or Windows. (This is true under Windows NT, but under Windows 95 printer setup works normally).

\section{Window}

The standard menu commands are available. However, the only useful function item is the list of current windows. Use these to switch between the text and graphics window. The text window contains a measurement summary. 
Display

\section{Single Dataset}

This display option, the default option, displays the current measurement or catalog. The other display options are File Comparion, Ratio to Reference, and Ratio to Calibration. The best way to switch between these options is to always revert to Single Dataset before picking any other option.

\section{File Comparison}

The prompts the user to load another measurement to be compared with the current measurement. When this option is used, all plots (except multiplicity) will display both measurements. (Because of the way the original software was written, multiplicities are not shown under this option). This command is a convenient way to show two measurements together, but measurement catalogs can be used for the same purpose and will show any plot.

\section{Ratio to Reference}

This command loads a measurement or catalog of measurements that are used as a "reference measurement". Loading a reference enables signature matching. The plots become to ratio plots, in which the ratio of the unknown (currently loaded measurement) to the reference measurement is shown. If the reference is a catalog, the plots also show upper and lower bounds for the ratio. These bounds reflect the variation of the ratio among the reference measurements, which establishes the acceptable bounds of the ratio. (The $X$ axis of the plots might also be randomly scrambled so that the frequency dependence of the ratio is disguised).

When IDAS loads a single measurement as the reference the user is warned that "class deviation is set to value". This cryptic message is just a reminder that IDAS cannot determine the expected variation of the reference signal if it has only one example of a reference measurement.

\section{Negative Log Ratio}

This command shows the negative log of the ratio. This indicates the change in the "scattering" cross section between two measurements. Once selected it is checked; selecting it again resets this display option.

\section{Randomized Ratio}

This command scrambles the $X$ axis of the ratio display. Once selected it is checked; selecting it again resets this display option.

\section{Ratio to Calibration}

A calibration is an active measurement performed in air (no target), with the same detector configuration as a measurement with the target. If a measurement catalog has been loaded, and that catalog contains calibrations for each measurement in the catalog, this command displays the ratio of the measurement to its calibration. 
Negative Log Ratio

This indicates the change in "scattering" cross-section between an air measurement (no container) and the container/component measurement. This is mainly used to display source-detector correlations. This option appears twice in the pull down menu for obscure reasons.

Catalog

Create Catalog

The user is prompted to create a catalog of measurements. First, a standard "file open dialog" is used to select multiple measurements to appear in the catalog. Second, a standard "file write dialog" is used to select the file name of the catalog file. IDAS adds this new catalog to the "most recently used" list of the File menu so that it can be opened easily. (A catalog creation command is also available if no measurements are loaded. In this case, IDAS offers to load the catalog immediately).

\section{Single Measurement}

This option is currently disabled. Previously, if a catalog were loaded, all measurements would normally be plotted. This command would let the user select a single measurement for display.

\section{All Measurements}

This option is currently disabled. Previously, this command restored the display of all measurements in the catalog.

Caution: some commands will automatically turn off this option and change the selection the first measurement in the catalog. This had been done to protect the graphics functions from a situation in which both a measurement catalog and reference catalog was loaded. This might not be necessary any longer, however some testing would need to be done to prove it.

\section{Select Measurements}

This command opens a dialog box from which a subset of the catalog measurements are selected for plots, etc.

Analysis

\section{Detector Efficiency}

This command analyzes and displays detector efficiency as a function of energy. This requires that an active measurement be loaded.

The function offers to write results into the file efficiency.csv. The format of this spreadsheet file is the measurement name followed by the detector thresholds and peak efficiencies for all detectors. (The threshold and peak efficiency values are calculated by fitting a model to the detector data.) This file is useful in documenting the detector calibration over the a series of measurements. 
The function offers to plot the fitted detector efficiency curves with

The user first specifies some parameters:

Detector sizes are specified by as a circular diameter, in centimeters (this really should be edge length since most detectors are square);

Distance between the source and each detector in centimeters;

Location of the gamma peak in the source-detector cross correlation, in nanoseconds, with the default value being where IDAS has located the peak;

Lowest energy to analyze and plot in MeV.

Highest energy to analyze and plot in MeV.

\section{Detector Efficiency Parameters}

Det 1

Detector Diameter (cm)

Source/Detector

Distance (cm)

Gamma Peak

Location (ns)

Low Energy

CutOff (MeV)

High Energy

CutOff (MeV)

$\sqrt{10.72}$

\section{2}

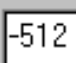

0.4

6
Det 2

$\longdiv { 1 0 . 7 2 }$

104.9

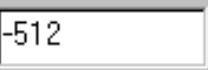

0.4

6
Det 3

$\longdiv { 1 0 . 7 2 }$

\section{9}

\section{$-512$}

0.4

6

Det 4

10.72

106.2

$-512$

0.4

6
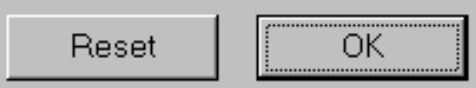

Cancel

If a catalog is loaded and all measurements are selected for display, IDAS offers to plot efficiency for the entire catalog of measurements. Otherwise it plots efficiency for a single measurement. If one measurement is analyzed, the user is given the option of plotting the efficiency on one graph or separate graphs.

This function would be more convenient if there were a simple way to set all detectors at once (usually the detector are identical). IDAS sets the default values to the user's last settings, however these settings are not kept when IDAS is restarted; they should be saved in the registry with other settings which IDAS keeps.

\section{Use Calibration}

This command should not be used. It sets a flag that indicates that the calibration for each measurement should be used in certain functions. However this flag is currently ignored. 


\section{Find Gamma Counts}

This command currently has no effect. At one time this command analyzed the measurement and located the gamma peak in the source-detector cross correlations. This is currently done automatically as IDAS loads the measurement. The menu item remains because it might be used to perform an interactive search for the gamma and neutron peaks, allowing the user to override IDAS as necessary.

\section{Correlation Options}

When IDAS loads a measurement it reads the correlations and multiplicities (PDFs) from the measurement files DAUI creates. The correlations data read from the file are the number of counts observed at each correlation lag. When IDAS reads these correlations it immediately processes them according to the options described in this section, then computes the spectra from the processed correlations. The processing options are specified according to the dialog box below. This is the same dialog that pops up when IDAS is started.

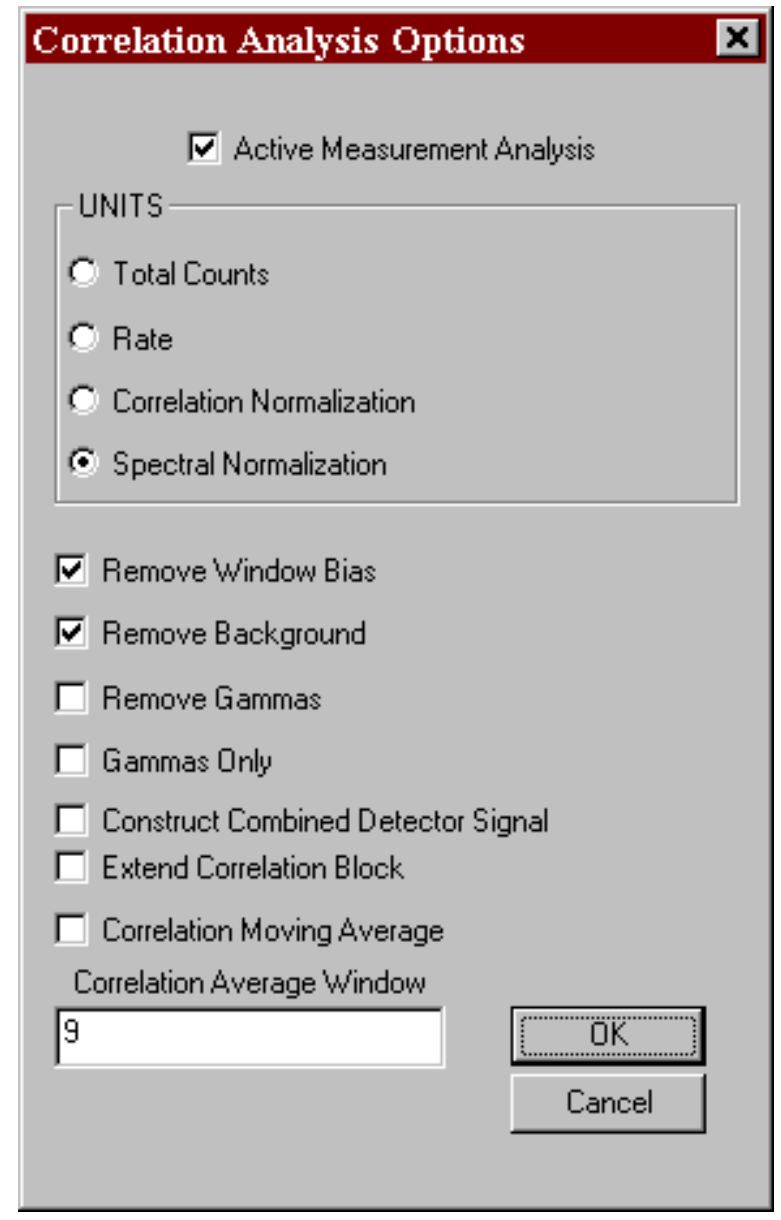

The Active Measurement Analysis check box should be checked whenever an active measurement is loaded. IDAS uses this information to verify the user's other processing selections. This is necessary because some correlation units and processing are not 
possible for passive measurements. This determination is normally made from the measurement data, however it can be overridden by the user.

Active analysis assumes that the $\mathrm{Cf}$ source is signal 1 (first channel); passive analysis assumes there is no $\mathrm{Cf}$ source. Units that normalize by the $\mathrm{Cf}$ count rate are not possible for passive analysis. The processing options which require locating the gamma and neutron peaks in the detector signals are possible only for active analyses.

IDAS processes all loaded measurements in the same way. Changing any of these options will cause all measurements to be reprocessed, including reference measurements and the comparison measurement. One consequence of this is that the active/passive analysis flag is set the same for all measurements. When the data file format is revised to contain the active/passive flag, this will no longer be true.

Units options are:

Total Counts simply shows the total number of auto/cross correlation counts collected during the measurement at each correlation lag.

Rate is primarily a passive measurement normalization. It is the total counts divided by the total time and the sampling interval, indicating "correlated" counts per second which occur per nanosecond of sampling (correlation) time. The spectral units that result from this option are counts per second.

Correlation Normalization is an active measurement normalization. It is useful when comparing the correlations of two measurements performed at different sampling rates, but is not generally used. The total counts are divided by the number of Cf fissions and the sampling interval ("correlated" counts per Cf fission per nanosecond of sample window time), except lag 0 of the autocorrelations, which is divided only by the number of $\mathrm{Cf}$ fissions (counts per $\mathrm{Cf}$ fission). The spectra are not used when this option is selected.

Spectral Normalization is the normalization normally used for active measurements. The total counts at each lag are divided by the number of Cf fissions and the sampling interval ("correlated" counts per Cf fission per nanosecond of sample window time). The spectral units that result from this option are counts per Cf fission.

Basic processing options are:

Remove Window Bias compensates for a bias introduced by the fact that the larger lags are averages over shorter times than the smaller lags, and should be selected when random signals are measured;

Remove Background subtracts the incidental correlations.

Some experimental processing options remove counts in source-detector correlations:

Remove Gammas simply removes all correlation counts under the gamma peak;

Gammas Only removes all correlation counts except those under the gamma peak. Note that gamma counts which appear outside the gamma arrival peak are left in as it is not possible to distinguish them from neutron counts. 
The detector autocorrelations are also adjusted by these options, but it is not possible to adjust the source auto correlation and detector cross correlations. As a result, the correlations are left in an inconsistent state.

Construct Combined Detector Signal is an experimental option that will combine detector signals together to make a pseudo-detector signal, provided that only four or less signals were acquired. This option is not fully implemented and should be used with caution.

Extend Correlation Block is an experimental option which artificially extends the correlation measurement to the largest data block size IDAS can process. This is not typically used.

Correlation Moving Average simply averages adjacent correlation lags together to smooth the measurement for plotting. The spectra are also computed from the smooth correlations. This is not typically used, but is useful when plotting many measurements with poorly converged correlations. The Correlation Averaging Window is the number of lags to average together for this option. This number is typically an odd integer $(1,3,5$, etc) so that the averaged lags include the central lag and the ( $\mathrm{N}-1) / 2$ lags on either side.

\section{Multiplicity Options}

IDAS processes multiplicities when it opens a measurement file. The Multiplicity Options dialog pops up when IDAS starts, and is also available through this command.

DAUI now measures a different set of multiplicities. The IDAS GUI is only partially adapted to these changes: unused GUI controls are disabled but not yet removed.

Some processing options require the use of a random signal distribution. The options below refer to this as the "random multiplicity". This PDF is calculated by IDAS for each signal, and is the PDF which would result if (1) the signal were Poisson random, (2) had the average count rate measured for the signal, and (3) had the channel dead time measured for that signal. (The channel dead time is measured from the signal's autocorrelation).

These options are applied to the individual and combined detector signals.

The units are:

Total Blocks shows the total number of data blocks for each multiplicity value;

Fraction of Blocks shows the fraction of data blocks;

Normalized by Expected Random divides the number of data blocks by the number of blocks that a Poisson random source would have generated;

Normalized by Multiplicity Singles divides the measured multiplicity by the number of multiplicity(1) blocks observed.

The two units that are generally useful are total-blocks and fraction-of-blocks (the normal selection). The other units are experimental values that are sometimes used when experimenting with analysis. Regardless of this selection, the multiplicity moments are calculated in terms of probability (fraction of blocks). 
The option Dead Time Correction specifies that the calculated Poisson random PDF should account for the channel dead time; otherwise a straightforward Poisson PDF is used.

The option Subtract Random Multiplicity specifies that the calculated Poisson random PDF be subtracted from the measured PDF.

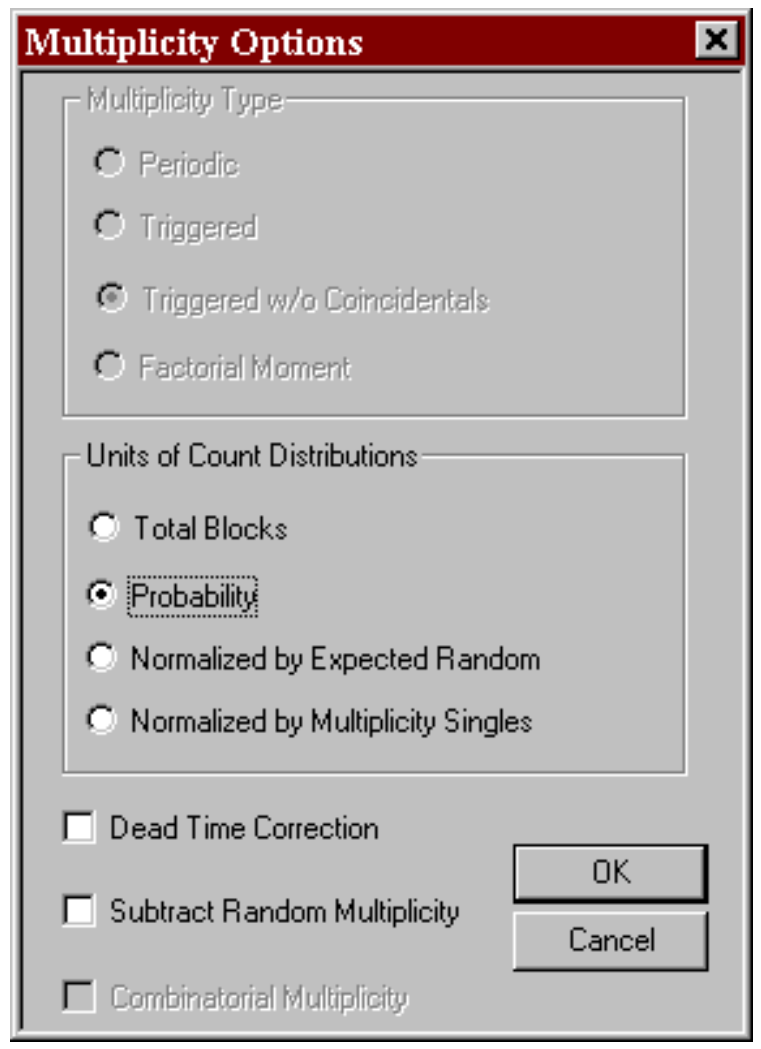

Multiplicity Analysis

This command is not implemented. A future version may produce a report for a detailed analysis of multiplicity.

\section{Write Measurement as CSV}

This command is nearly identical to the Write Measurement as Text command. The text file is written in the "comma separated value" (CSV) format. The advantage is that this format can be read by spreadsheets. If a catalog is loaded, this option offers to write all of the measurements as a text file. The file extension of these files is "CSV". The spectra are not written if the user elects to write unprocessed data (IDAS's processing creates the spectra). If the spectra resulting from unprocessed data are required, which is unlikely, the user can select those correlation and PDF options which do not change the data, and then write the "processed" data with this command.

\section{Write Measurements Report}

This command writes a small report on the measurement. This is not frequently used, but the purpose of the report is to summarize some important aspects of the data. This report could be placed in a report or log book. The report contains a line, written in CSV format, 
which contains the information most often used. This line would be used if one were creating a spreadsheet that summarized all of the data available.

\section{Write Measurements Stats to CSV}

This command writes measurement statistics to a comma-separated-value (CSV) file. It is a quick means of writing the observed mean and standard deviations of the signatures for a catalog of measurements. It also writes the theortical standard deviations due to the randomness of the signals. The latter should be smaller than the observed standard deviation since the observed signal includes additional sources of error however, by chance, the observed standard deviation can be smaller.

\section{Combine Measurements}

This command combines measurements to create a new measurement file. (This command is also available before IDAS has loaded any measurement). It operates much the same as catalog creation. The user selects the measurements to be combined, either by selecting all measurements in the current catalog or by selected multiple PAR files in a standard "file open" dialog box. The user then selects the file name for the combined measurement file. IDAS examines the individual measurements to make sure they are compatible: for example, measurements made at different sampling rates cannot be combined. IDAS also creates a TXT file that lists the measurements combined in the number of data blocks in each.

\section{Matching}

Matching uses a subset of the available signals and signatures. The following commands are used to select a subset of signals and signatures: if the signal is selected AND the signature is selected, that signature for that signal is used.

Matching commands are not enabled until a reference measurement or catalog is loaded (menu command DisplaylRatio to Reference).

\section{Select Signals}

This command brings up a dialog in which the user selects signals to be matched.

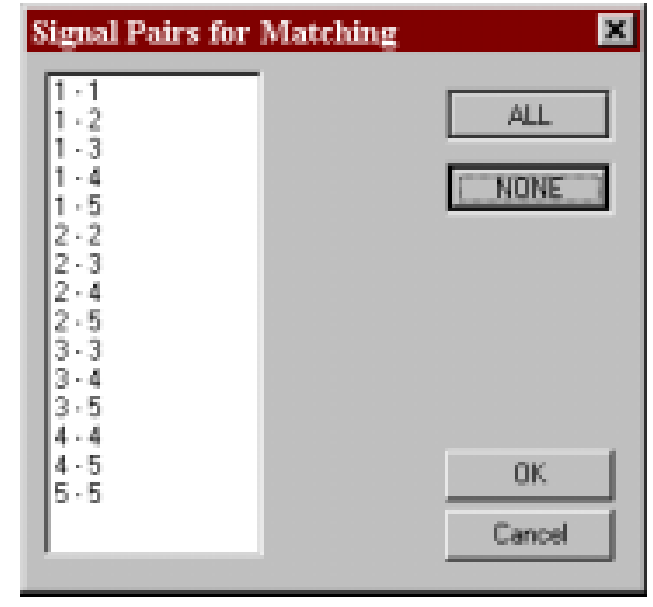


The signals are not selected individually but by signal pairs. For example, selecting "1-2" enables matching for all signatures computed from both signals 1 and 2 (cross correlation $1-2$, cross spectra 1-2, etc). Selecting $1-1,2-2,3-3,4-4$, or $5-5$ enables matching for the auto correlations, spectra, and PDFs of the individual signals. The combined detector PDF, which uses all of the detector signals, is selected by the Select Features dialog (below).

Matching is not automatically recalculated when the signal selection is changed.

\section{Select Features}

IDAS uses the word "features" to mean values derived from the measurement data which are used in the matching (this is standard pattern recognition terminology). The words "measurement" and "signatures" are often used to mean the same thing since a feature is usually a correlation, spectrum, or multiplicity.

This command brings up a dialog box used to select the features IDAS can use. The illustration shows a typical selection.

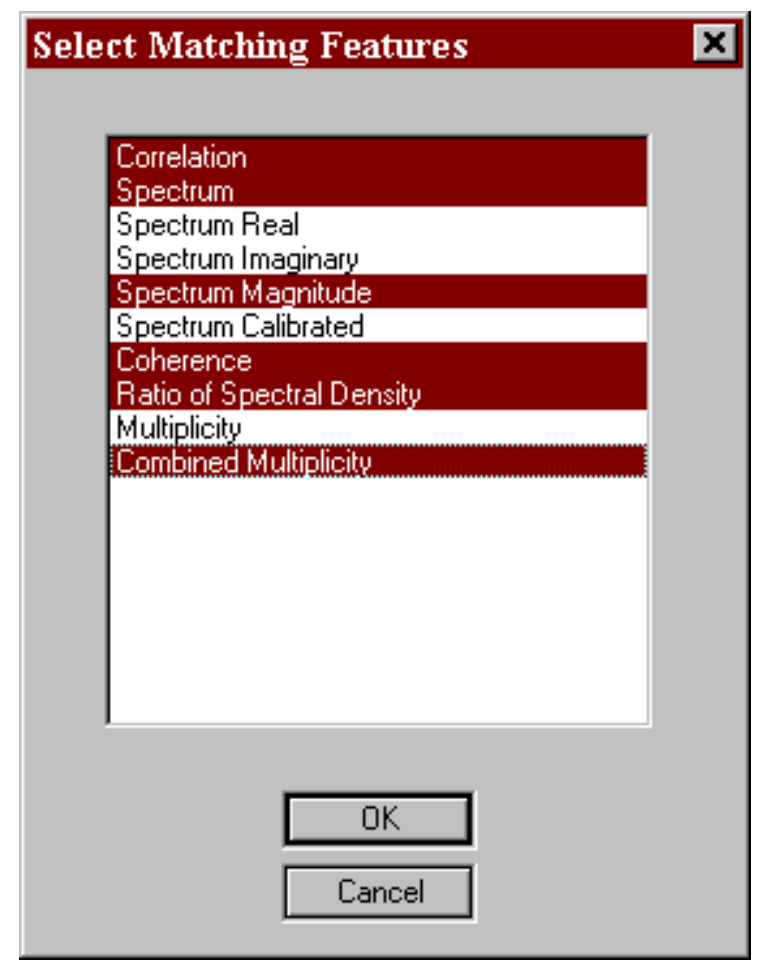

The options are:

Correlation selects auto and cross correlations;

Spectrum selects the auto and cross spectra, complex-valued for cross and realvalued for auto spectra; 
Spectrum Real and Spectrum Imaginary select the real and imaginary parts of the spectra, matched separately (experimental);

Spectrum Magnitude selects the magnitudes of the auto and cross spectra instead of the complex values;

Spectrum Calibrated is currently ignored (as are all "calibrated measurement" options);

Coherence selects the coherences;

Ratio of Spectral Density selects the special spectral quantity of the same name;

Multiplicity selects the "best" multiplicity or PDF available for an individual signal. The best value is the periodically-triggered PDF for older measurements and the calculated active/passive multiplet PDF for more recent measurements. For the recent measurements the measured multiplicities have a trigger signal and an event (counting) signal. This feature option selects each individual signal (see Select Signals command) as a trigger and the combined detectors signal as the event signal. The multiplicity moment, which is what is usually meant by multiplicity, is never selected (will be available in some future version);

Combined Multiplicity selects the "best multiplicity signature" of the combined detector signal. The is the periodically-triggered PDF of the combined detector signal for older measurements and the active (source-triggered) or passive (self-triggered) multiplets PDF for newer measurements.

Matching is not automatically recalculated when the signature selection is changed.

\section{Select Baseline}

This command is enabled when the reference is a catalog of measurements. It selects a particular measurement in the reference catalog as the baseline (prototypical) value of the reference. The alternative is that the average of the reference catalog defines the baseline value. The Add and Delete buttons have no effect.

\section{Options}

This set of options determines how the matching is performed. Many of these options were carried over from another computer program that was used to experiment with matching. Some options of these options have not been re-implemented in IDAS. These unimplemented options are in the Min Corr Count Option and Time Shift Method lists. 


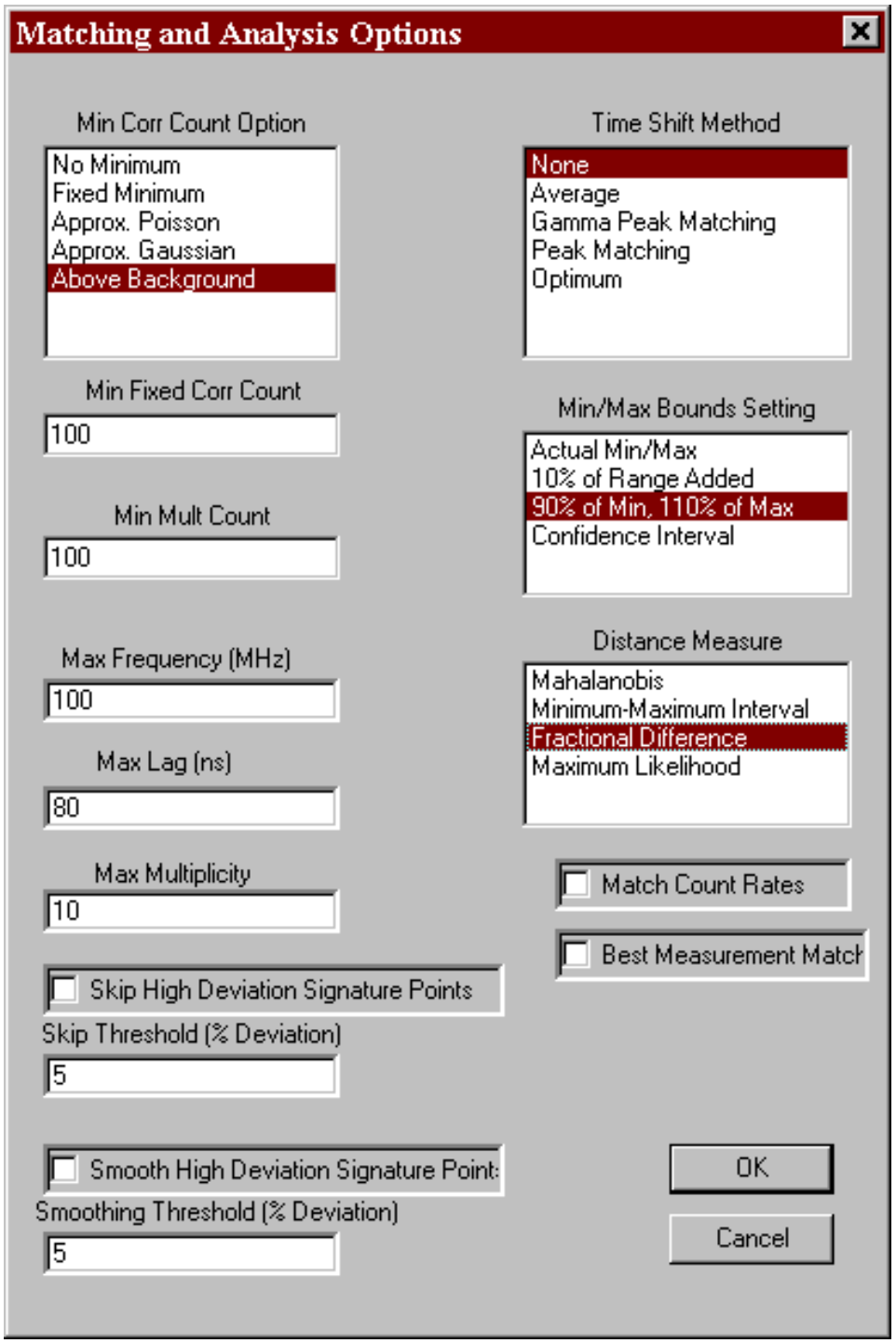

Min Corr Count Option specifies how to decide to ignore some regions of the correlations. If the measured correlation value at some lag has very few counts it has a high degree of uncertainty. Almost all correlations will have few counts a the longest lags. The options are:

No Minimum specifies that any number of counts is acceptable, and should never be used;

Fixed Minimum specifies that the number the user enters into the Min Fixed Corr Count window is the lower limit, and these must be counts above the incidental (background) count level;

Approx. Poisson (not implemented) specifies that there should be enough counts to approximate a Poisson distribution;

Approx. Gaussian (not implemented) specifies that there should be enough counts to approximate a Gaussian distribution; 
Above Background specifies that the number of counts should be above the incidental correlation count value.

A good option to use is Fixed Minimum with a Fixed Count value of at least 100. This insures reasonable statistical accuracy.

Min Mult Count specifies the minimum count multiplicity/PDF value (units of data blocks observed) required for the value to be used in matching. For example, if the minimum value specified were 100 and 99 blocks were observed with 8 counts in them, PDF(8) would not be used in matching.

Max Frequency $(\mathrm{MHz})$ specifies the maximum frequency to match in spectral signatures. This option does not guarantee that all frequencies below this value will be used since these lower frequencies might be dropped due to other criteria (e.g., poor statistical accuracy).

Max Lag (ns) specifies the maximum correlation lag to match. Both positive and negative lags up to this value are included. Other criteria are typically used to drop lags with poor matching properties (Min Corr Count).

Max Multiplicity specifies the highest count PDF to use. Other criteria can be used to drop PDFs with poor matching properties.

Skip High Deviation Signature Points specifies that signature points (frequencies, lags, and PDFs) should not be used if the reference signature has poor statistical accuracy. The accuracy criteria is set by the user in the edit box below, Skip Threshold (\% Deviation). The best threshold depends on the analysis being done. Low thresholds can give more consistent matching results since the less accurate points of the reference signatures are skipped, but lower thresholds can also cause matching to skip points of the signatures which are useful in discriminating between components.

Smooth High Deviation Signature Points is an option that has not been implemented yet. Likewise, the associated option Smoothing Threshold (\% Deviation) has not been implemented.

The Time Shift Method is retained from the previous software. IDAS does not apply a time shift to any signals while matching; leave "None" selected.

Distance Measure specifies how the match distance between the unknown and reference signature(s) is calculated:

Mahalanobis specifies the Mahalanobis distance measurement. This distance measurement is the difference between the unknown and reference values in units of standard deviation of the reference. The standard deviations of the reference signatures calculated by IDAS from the deviations among the measurements in the reference catalog. If only a single reference measurement is available, another distance measurement must be used. Also, if very few reference measurements are available, the standard deviation cannot be calculated accurately.

Minimum-Maximum Interval specifies that a signature point matches if the unknown's point lies within some interval about the reference point. The interval is specified by the Min/Max Bounds Setting option box (above the Distance Measure box). Points that match are assigned a distance of 0 , and points that do not match are assigned a 
distance of 1 . This option is not frequently used and may not be completely debugged.

Fractional Difference specifies that the distance measurement calculated as a fraction (percentage) of the reference signature point's value. For example, if the reference's CPSD12 value is 100 at $30 \mathrm{MHz}$ and the unknown's is 90, the distance is (100$90) / 100=0.1$. This distance is used often, especially when onlly one reference measurement is available.

The Maximum Likelihood option has not been implemented in IDAS.

Min/Max Bounds Setting specifies the nature of the match/mis-match boundry used for the Minimum-Maximum Interval distance measure above:

Actual Min/Max specifies that the matching bounds is the minimum and maximum reference signature values for each point. This option is not likely to yield good results unless a very large number of reference signatures are used because of the large number of signatures points (often 700). The probability of a few (10) reference measurements containing the minimum and maximum signature values of 700 points is very small.

$10 \%$ of Range Added specifies than the observed minimum-maximum interval be extended by $10 \%$. This option would be used when enough references are available to establish good statistical bounds for the signatures.

$90 \%$ of Min, $110 \%$ of Max specifies that the bounds be set to the $90 \%$ of the minimum observed and $110 \%$ of the maximum observed. This option uses an assumption, often true, that the measurements are accurate to $10 \%$ and any signature point within that interval should be accepted.

Confidence Interval specifies that the bounds should be extended according to the number of reference measurements used. IDAS calculates the standard deviation of each reference signature point, then increases the standard deviation according to how many reference signatures were available to calculate the standard deviation. IDAS uses a standard statistical formula for the uncertainty in the calculated standard deviation to increase the interval enough that it is has a 99\% probability of being as large as the true interval (if the references are truly representative measurements).

Match Count Rates specifies that IDAS should first apply a gain to each signal which equalizes that signal's count rate for the unknown and reference measurement. This will cause the auto spectra/correlations and multiplicities to match exactly if they have the same shape with respect to frequency/lag. It may cause the cross spectra/correlations to match well, however the two gains applied to the cross signatures (one gain from each of the two signals). If the unknown matches the reference, these equalizing gains will remove some of the effects of mis-positioning detectors. This option is used to help identify a "detector gain" problem.

Best Measurement Match specifies that IDAS should match each unknown to each measurement in the reference catalog, and find the reference it matches best (smallest overall match distance). Otherwise, IDAS matches the unknown to the average of the references in the catalog. This option is especially useful when each reference is a measurement of a different component; in this case the most appropriate match distance measure is Fractional Difference. 


\section{Do Match}

This command performs the matching between the references (load by the command Display | Ratio to Reference) and the unknowns (the currently displayed measurements). The options described above are used. The matching result is not automatically displayed or written to a file, but can be plotted (see the Plots menu command).

\section{Match All and Write CSV}

This option matches each unknown to each reference and writes the results to a commaseparated-value (CSV) file that a spreadsheet program can read. It is the equivalent of selecting each reference individually and matching to it, ignoring all other refernences loaded in the reference catalog. The appropriate Distance Measure is Fractional Difference since there is only one reference. It differs from other matching options in that the matching results are not stored, but are instead written to a CSV file.

The user is asked if detailed and/or summary match results are desired, and the name of the corresponding CSV file.

The detailed results CSV file contains all unknown-reference comparisons in the following order:

1. summary table of all unknowns to reference \#1,

2. detailed table comparing unknown \#1 to reference \#1,

3. detailed table comparing unknown \#2 to reference \#1,

4. and so forth for the rest of the unknowns compared to reference \#1,

5. repeat parts $1-4$ for the second reference,

6. and so forth for the rest of the references

A typical summary table is show below, for the case of matching one unknown (ki2805) to a reference. Only the overall match distance is shown in the summary table.

\begin{tabular}{|l|l|l|l|l|l|l|}
\hline unknown & signature & mean & rms & min & max & points \\
\hline ki2805 & Overall & $2.96 \mathrm{E}-01$ & $8.05 \mathrm{E}-01$ & $-9.94 \mathrm{E}-01$ & $1.48 \mathrm{E}+01$ & 1461 \\
& & & & & & \\
\hline
\end{tabular}

The detailed comparison has the format show below. The first three lines provide some general information about the measurements being compared: the measurement file name, the catalog it was loaded with (if any), number of signals, sampling rate, block size, and number of blocks. The remaining lines contain the signature comparisons: the signature name, its mean (average) match distance, variance of the match distance (described below), minimum and maximum match distance in the signature, and the number of signature points (frequencies, lags, etc) of the signature used. The variance is calculated as: 


$$
\sqrt{\frac{1}{N}{ }_{i=1}^{N} D_{i}^{2}}
$$

where $\mathrm{N}=$ the number of points in the signature and $\mathrm{D}_{\mathrm{i}}=$ the match distance at point $\mathrm{i}$ of the signature.

The first signatures listed are "overall" signatures. The overall signatures combine all of the individual signatures of a type. For example, the "Corr Overall" signature combines all correlation signatures together. The "Overall" signature combines the individual signatures of all types together. IDAS decides which reference best matches an unknown measurement by using the "Overall" value.

The CSV file format does not vary so that these files are easy to use in spreadsheets. To maintain this format, signatures that are not matched are nevertheless written to the CSV file. Unused signatures are indicated by number of points used (the last column), which will be 0 ; the mean, variance, minimum, and maximum values are set to 0 .

\begin{tabular}{|c|c|c|c|c|c|}
\hline measurement & Catalog & signals & sample rate & block size & blocks \\
\hline ki2805 & ki-poly & 3 & $1.00 \mathrm{E}+09$ & 512 & $2.00 \mathrm{E}+08$ \\
\hline ki2804 & ki-poly & 3 & $1.00 \mathrm{E}+09$ & 512 & $2.00 \mathrm{E}+08$ \\
\hline sig & mean & var & $\min$ & $\max$ & meas \\
\hline Overall & $2.96 \mathrm{E}-01$ & $8.05 \mathrm{E}-01$ & $-9.94 \mathrm{E}-01$ & $1.48 \mathrm{E}+01$ & 1461 \\
\hline Corr Overall & 6.87E-01 & $1.28 \mathrm{E}+00$ & $-5.38 E-01$ & $5.45 \mathrm{E}+00$ & 261 \\
\hline Spec Overall & $3.34 \mathrm{E}-01$ & $5.41 \mathrm{E}-01$ & $2.57 \mathrm{E}-06$ & $4.59 \mathrm{E}+00$ & 300 \\
\hline Spec Re Overall & $2.49 \mathrm{E}-01$ & 5.07E-01 & $\mid-3.65 \mathrm{E}-01$ & $4.53 \mathrm{E}+00$ & 300 \\
\hline Spec Im Overall & $-1.20 \mathrm{E}-01$ & $2.64 \mathrm{E}-01$ & -8.71E-01 & $3.72 \mathrm{E}-01$ & 150 \\
\hline SpecMag Overall & 2.57E-01 & 5.03E-01 & $-9.19 E-01$ & $4.45 \mathrm{E}+00$ & 300 \\
\hline Coh Overall & $1.25 \mathrm{E}-01$ & $1.34 \mathrm{E}+00$ & $-9.94 E-01$ & $1.48 \mathrm{E}+01$ & 150 \\
\hline SpcRatio Overall & $0.00 \mathrm{E}+00$ & $0.00 \mathrm{E}+00$ & $0.00 \mathrm{E}+00$ & $0.00 \mathrm{E}+00$ & 0 \\
\hline Mult Overall & $0.00 \mathrm{E}+00$ & $0.00 \mathrm{E}+00$ & $0.00 \mathrm{E}+00$ & $0.00 \mathrm{E}+00$ & \\
\hline Corr $[1,1]$ & $0.00 \mathrm{E}+00$ & $0.00 \mathrm{E}+00$ & $0.00 \mathrm{E}+00$ & $0.00 \mathrm{E}+00$ & 1 \\
\hline Corr[1,2] & $1.28 \mathrm{E}+00$ & $1.78 \mathrm{E}+00$ & $-4.27 \mathrm{E}-01$ & $5.45 \mathrm{E}+00$ & 82 \\
\hline$\ldots$ & $\cdots$ & $\ldots$ & $\ldots$ & $\ldots$ & $\ldots$ \\
\hline Mult[5] & $0.00 \mathrm{E}+00$ & $0.00 \mathrm{E}+00$ & $0.00 \mathrm{E}+00$ & $0.00 \mathrm{E}+00$ & 0 \\
\hline Comb Mult & $0.00 \mathrm{E}+00$ & $0.00 \mathrm{E}+00$ & $0.00 \mathrm{E}+00$ & $0.00 \mathrm{E}+00$ & 0 \\
\hline
\end{tabular}

The summary results file contains a table with the following format.

\begin{tabular}{|l|l|l|l|l|l|l|}
\hline & ref pts & UNK-1 & UNK-2 & & UNK-1 & UNK-2 \\
\hline REF-1 & 103 & 26.4 & 26 & REF-1 & 6.2 & -1.2 \\
\hline REF-2 & 103 & 41.7 & 45.8 & REF-2 & 3.8 & -1.8 \\
\hline REF-3 & 103 & 39.4 & 29.8 & REF-3 & 0.6 & -7.1 \\
\hline
\end{tabular}


This table shows the overall match distance between each unknown (UNK) and reference signature (REF). The first column is the number of signature points used in the match. Each unknown's match RMS distance appears in the following columns. The next column repeats the reference signature names; each unknown's average match distance appears in the following columns.

\section{Show Unknown Ranking}

This option is not currently implemented.

\section{Write Match Report}

The current matching results are written to a text file the user selects. The report file contains the same matching information as the CSV file, and also lists the signal and matching options used (in effect when the report is written).

\section{Write Match CSV}

The current matching results are written to a CSV file the user selects. The CSV match file contents are described above.

\section{Write Count Rate Analysis}

This command performs a match based on "total correlated count rate" and writes the results to a text file the user selects.

The correlated count rate is calculated by subtracting the incidental correlations from the total auto and cross correlations. For active measurements, this is normalized by the Cf fission rate. This is a measure of the overall correlation between two detectors (and is related to the $0 \mathrm{~Hz}$ value of the auto and cross spectra).

Each unknown measurement is matched to each reference measurement. The results file contains a series of tables, each showing the match of one unknown to all references. For example:

\section{Matching measurement KJ0123}

\begin{tabular}{|l|l|l|l|l|l|l|}
\hline Det & kj0106 & $\mathbf{k j 0 1 0 7}$ & $\mathbf{k j 0 1 0 8}$ & $\mathbf{k j 0 1 0 9}$ & $\mathbf{k j 0 1 1 0}$ & BEST \\
Pair & & & & & & \\
\hline $\mathbf{1 - 1}$ & 0.149 & 0.096 & 0.121 & 0.059 & 0.077 & $\mathrm{kj0110}$ \\
$\mathbf{1 - 2}$ & 8.648 & 3.643 & 2.438 & -3.387 & 1.406 & $\mathrm{kj0110}$ \\
$\mathbf{1 - 3}$ & 6.71 & 1.281 & 2.249 & -1.064 & 3.657 & $\mathrm{kj0109}$ \\
$\mathbf{2 - 2}$ & 19.864 & 8.119 & 8.658 & 5.426 & 11.319 & $\mathrm{kj0109}$ \\
$\mathbf{2 - 3}$ & 9.242 & 0.529 & -0.68 & 0.483 & -1.406 & $\mathrm{kj0109}$ \\
$\mathbf{3 - 3}$ & 17.687 & 9.342 & 10.134 & 10.034 & 13.227 & $\mathrm{kj0107}$ \\
\hline
\end{tabular}


The table shows the percent difference between the correlated counts rate of each detector pair and the corresponding detector pair of each reference. The last column shows the best matching reference; it can be considered as a vote for a match to that reference.

The file also contains similar tables produced for:

Correlated counts rate / incidental count rate: This has the effect of further normalizing the correlated count rate by the average count rate. This analysis is an experiment.

Correlated direct gamma count rate: For active measurements, this uses the rate of arrival of directly transmitted Cf fission gammas. This is a way to separate gamma effects from neutron effects.

Correlated gamma-neutron counts: For active measurements, this uses the rate of arrival of radiation during the time period when directly transmitted $\mathrm{Cf}$ neutrons are expected. More precisely, this includes all radiation except the directly transmitted Cf fission gammas. This time also includes gammas and target fission radiation.

Plots

\section{Plot Selections}

This command opens a dialog box from which the user selects the signatures to be displayed in the graphical window. The figure below shows a 3-channel measurement. The frequency, correlation, and multiplicity signatures are grouped from left to right. The signature names follow the format

$$
<\text { signature }><\text { signal } 1><\text { signal } 2><\text { signal } 3>\text { [ }<\text { qualifier }>\text { ] }
$$

The signature names are:

APSD auto power spectral density;

CPSD cross power spectral density, with qualifiers [MG] for magnitude, $[\mathrm{PH}]$ for phase, [RE] for real, [IM] for imaginary, and [PO] for polar;

$\mathrm{COH}$ coherence,

SR ratio of spectral densities, which uses 3 signals, and has qualifiers [MG] for magnitude and $[\mathrm{PH}]$ for phase;

AUTO auto correlations;

CROSS cross correlations with qualifiers $[\mathrm{P}]$ for positive lags and $[\mathrm{N}]$ for negative lags;

P-PDF periodically-triggered detector count probability distribution

T-PDF count-triggered detector count probability distribution

MULT multiplets probability distribution 
MULTx[T/D] multiplicity triples/doubles does not currently work

MOMEN multiplet count distribution factorial moment (multiplicity)

The currently selected signatures appear in the Selections list box.

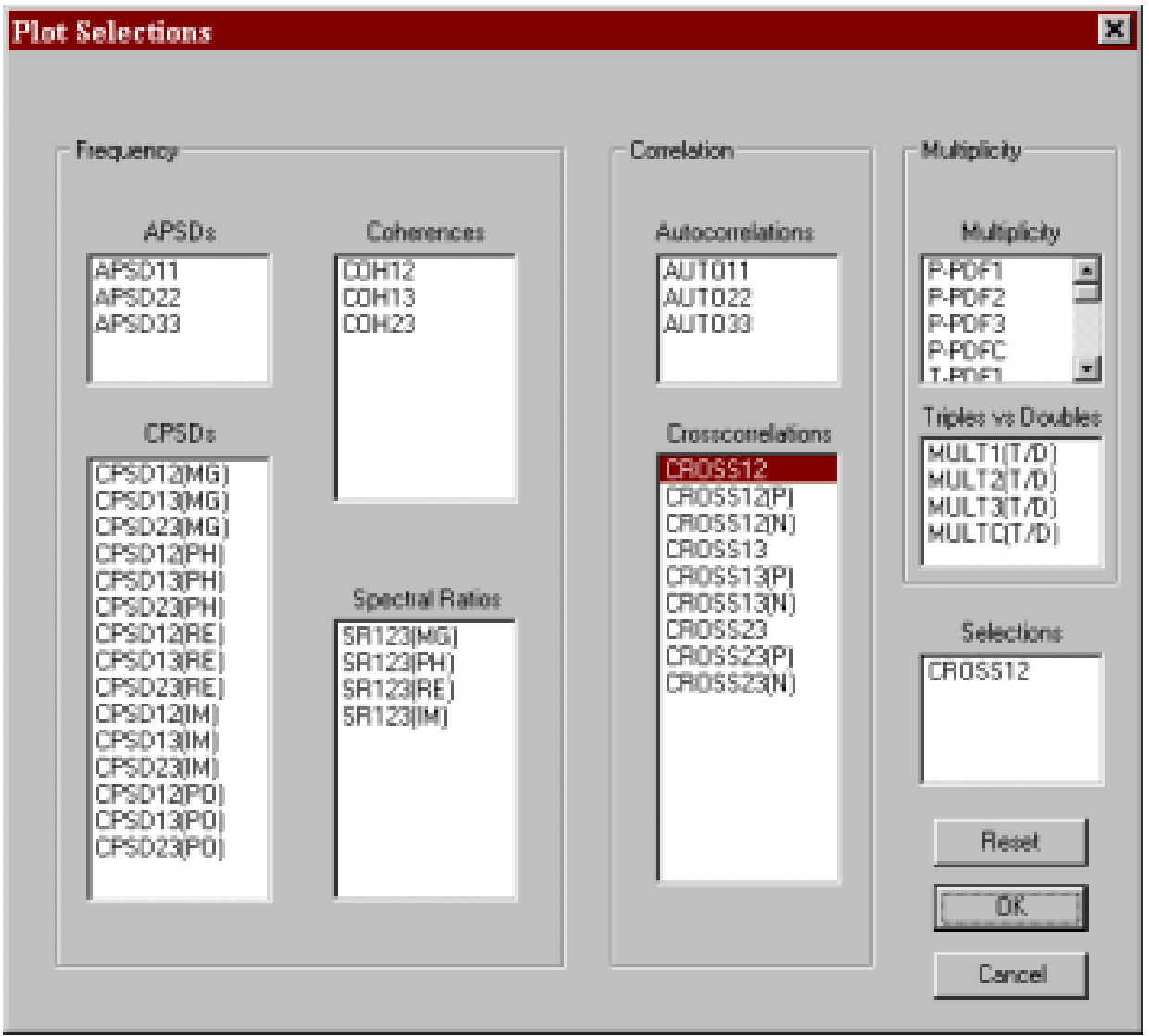

\section{Plot Settings}

This command opens the plot settings dialog box. This dialog controls the appearance of the plot. The Range Settings group sets the $x$ axis range to full, half, quarter, or $1 / 10$ of the full range. The User Input button activates a second dialog (Range Set, below) through which the user can set the $\mathrm{x}$ and $\mathrm{y}$ axis range directly. 

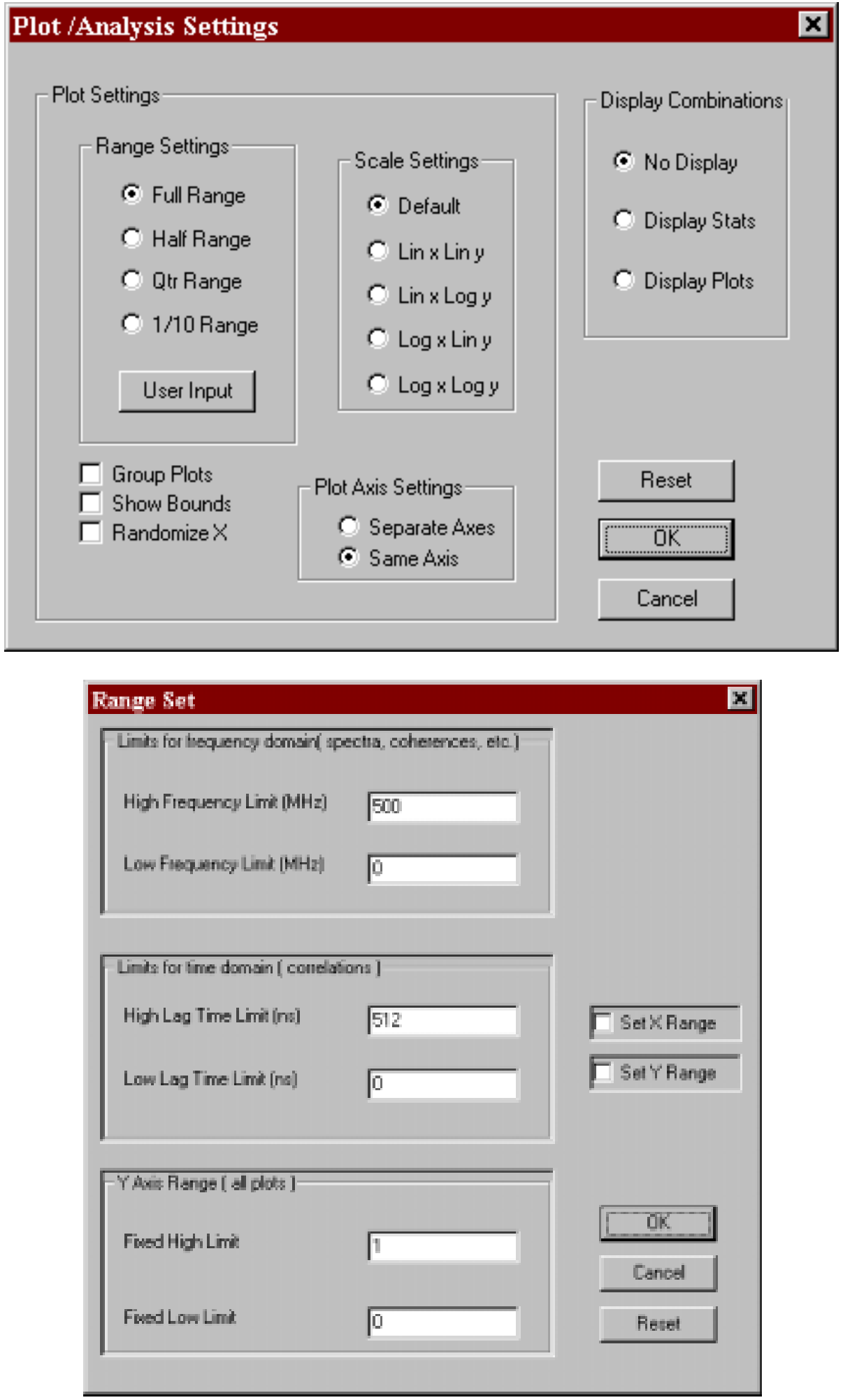

The Scale Settings group selects linear or log scaling for the $\mathrm{x}$ and $\mathrm{y}$ axises.

The Plot Axis Settings group determines whether curves are in one graph or multiple graphs on the page. The options are: each signature curve in a separate graph (Separate Axes) or all curves in one graph (Same Axis). 
Group Plots controls whether the entire measurement catalog is displayed, or one measurement from the catalog. It is a convenient way to select a full catalog display; if it is turned off only the first measurement in the catalog is selected for display. (The better way to select between catalog and single measurement plots is to use the Catalog menu on the main command bar).

Show Bounds controls whether the bounds of the reference measurements are shown with the measurement curve. Bounds can be shown when a reference catalog is loaded. Bounds for the ratio are shown if "ratio to reference" is selected, otherwise the actual reference bounds are shown.

Randomize $X$ controls whether the $\mathrm{x}$-axis is scrambled. $\mathrm{X}$ axis scrambling is used to show a signature without revealing secret information. Each time a new plot is selected the $x$ axis is scrambled in a new order. All curves shown are scrambled in the same way.

The Display Combinations group controls what plot information is shown in the IDAS text window. No Display selects no plot information, so the normal measurement summary information is displayed in the text window. Display Stats shows the average and standard deviation of the plotted curves. Display Plots shows the $\mathrm{x}$ and $\mathrm{y}$ values of the curves.

\section{Multiplicity Plot}

This command opens a dialog box through which periodically-triggered count PDF curves may be drawn. This command has been carried over from an old version and does not support the plotting options available through the Plots Selections dialog box. However, it plots the measured PDF and the calculated Poisson random multiplicity together. This is useful to quickly see how the detector PDF varies from a Poisson multiplicity.

The user specifies which signals to plot. The Det check box specifies the combined detector multiplicity. The Poisson model will incorporate signal dead time corrections if the Pulse Width Corrections box is checked. 


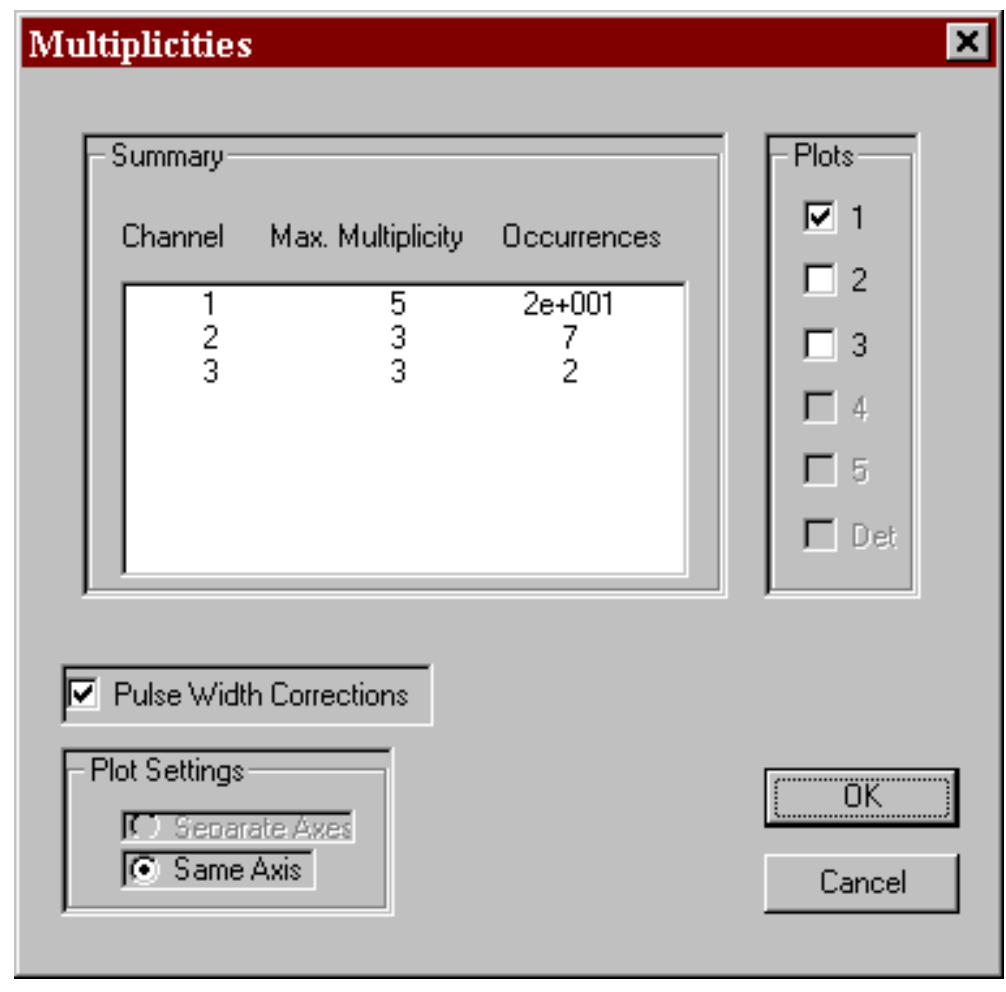

Plot Match Result

This command plots the distance between each unknown and the reference measurement, for a feature selected by the user. The following dialog box selects the feature. Both the mean distance and the variance of the distance are plotted for the signature. 


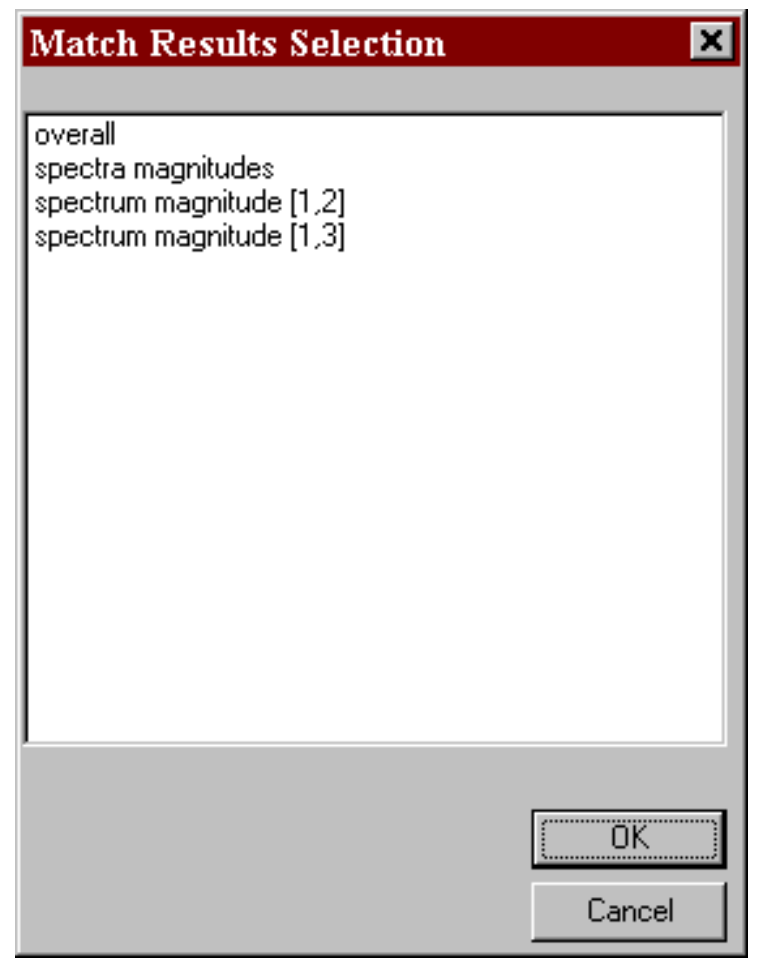

The dialog box lists only the features which were used in the matching.

\section{Plot Match Differences}

This command plots the distances between the unknown(s) and reference measurements for a feature selected by the user. The distance at each frequency / lag / multiplicity is shown. The feature is selected using the same dialog box as above.

\section{Print Plot for Catalog}

This command prints the current plot for each measurement in the catalog. One measurement is plotted on each graph; one graph is printed on each page. This command is used when a particular plot scale, range, etc., are desired and each measurement must be plotted on a separate graph.

\section{Print Archive Plots}

This command prints a preset group of plots. It can be used to print 'almost everything' in a measurement for an archive. The format is intended to minimize the amount of paper generated, and is not intended to produce graphs for detailed analysis.

If a catalog is loaded and All Measurements is selected, the entire catalog will be printed. The user is given the opportunity to use the current plot settings (ranges, etc.), otherwise a standard set are used. If a reference catalog is loaded, the reference bounds can be shown (see the Plot Settings dialog box). The standard set plots half of the range of most signatures (lower half of frequencies, etc.). 


\section{Print Match Plots}

This command prints all of the graphs available for the matching (see the Plot Match commands above).

\section{Test Randomization}

This command runs a long internal test of the scrambling of the $\mathrm{x}$ axis and writes some results to file Random.csv. It does not perform any analysis of data.

\section{REPLOT}

This command replots the current plot. Its only use is to redraw the plot when the window size changes.

Help

Help \About anwis1...

This command displays the version number of the software. 